\title{
Consequências nutricionais da privação de sono em crianças e adolescentes: uma
}

\section{revisão integrativa}

\author{
Nutritional consequences of sleep deprivation in children and adolescents: an integrative review \\ Consecuencias nutricionales de la privación del sueño en niños y adolescentes: una revisión
}

integradora

Recebido: 26/08/2021 | Revisado: 04/09/2021 | Aceito: 08/09/2021 | Publicado: 09/09/2021

\author{
Juliana Paiva de Oliveira \\ ORCID: https://orcid.org/0000-0003-0568-2058 \\ Centro Universitário UNA, Brasil \\ E-mail: julianapaiva05@gmail.com \\ Robson Vieira Ramos \\ ORCID: https://orcid.org/0000-0003-1851-5769 \\ Centro Universitário UNA, Brasil \\ E-mail: ramrobson@gmail.com \\ Angela Maria De Sousa \\ ORCID: https://orcid.org/0000-0001-5716-627X \\ Centro Universitário UNA, Brasil \\ E-mail: ams9818@yahoo.com.br \\ Fabiana Carla Teixeira De Oliveira \\ ORCID: https://orcid.org/0000-0002-6567-5514 \\ Centro Universitário UNA, Brasil \\ E-mail: fabyoliveiranutri@gmail.com \\ Nayara Ragi Baldoni \\ Centro Universitário UNA, Brasil \\ ORCID: https://orcid.org/0000-0002-3400-0725 \\ E-mail:nrbaldoni@gmail.com
}

\begin{abstract}
Resumo
O sono é um processo do corpo para regulação metabólica e fisiológica em todos os seres humanos, se tornando necessário tanto em sua quantidade e qualidade. Realizar uma revisão integrativa para analisar as consequências alimentares e nutricionais em crianças e adolescentes que sofrem de privação de sono. Efetuou-se uma busca sistematizada de artigos indexados nas bases de dados: PubMed, Biblioteca Virtual da Saúde (BVS) e Web of Science publicado até a data 03/12/2020. Foram incluídas publicações em todos os idiomas. Dois revisores independentes selecionaram os artigos elegíveis e extraíram os dados dos artigos selecionados. Com base nos 405 artigos encontrados, após aplicação dos critérios de inclusão, 28 artigos foram incluídos. Tendo em vista as consequências da privação de sono, foram encontrados fator de risco para sobrepeso e obesidade, maiores resultados de medidas antropométricas (circunferência da cintura, circunferência do quadril e percentual de gordura corporal), aumento de Índice Massa Corporal, maior consumo calórico, maior ingestão de alimentos industrializados e menor ingestão de frutas. Concluise que a privação de sono traz consequências negativas para crianças e adolescentes, tendo como um dos resultados principais o risco para sobrepeso e obesidade, maior ingestão de alimentos industrializados e mais calóricos, menor ingestão de frutas. Diante desses achados, educação em saúde sobre este tema deve ser mais discutida entre os pais e/ou responsáveis de crianças e adolescentes.
\end{abstract}

Palavras-chave: Privação do sono; Crianças; Adolescentes.

\begin{abstract}
Sleep is a body's process for metabolic and physiological regulation in all humans, becoming necessary both in it's quantity and quality. Conduct a integrative review in order to analyze the food and the nutritional consequences in children and adolescents suffering from sleep deprivation. A systematic search of articles indexed in the following databases was carried out: PubMed, Biblioteca Virtual da Saúde (BVS) and Web of Science published until 03/12/2020. Publications in all languages were included. Two independent reviewers selected the eligible articles and extracted data from those selected articles. Based on the 405 found, after applying the inclusion criteria, 28 articles were included. In view of the consequences of sleep deprivation, a risk factor for overweight and obesity was found, larger anthropometric measurements (waist circumference, hip circumference and body fat percentage), increase of Body Mass Index, higher caloric consumption, higher intake of processed food and lower intake of fruits. In conclusion, sleep deprivation has negative consequences for both children and adolescents, having as one of the main results the risk for overweight and obesity, higher consumption of ultra-processed foods, higher calorie intake and lower intake of fruits. Concludes
\end{abstract}


Therefore, health education on this topic should be further discussed among parents and/or guardians of children and adolescents.

Keywords: Sleep deprivation; Children; Adolescents.

\begin{abstract}
Resumen
El sueño es un proceso corporal de regulación metabólica y fisiológica en todos los seres humanos, por lo que es necesario tanto en su cantidad como en su calidad. Realizar una revisión integradora para analizar las consecuencias alimentarias y nutricionales en niños y adolescentes que sufren privación del sueño. Se realizó una búsqueda sistemática de artículos indexados en las siguientes bases de datos: PubMed, Virtual Health Library (BVS) y Web of Science publicados hasta el 03/12/2020. Se incluyeron publicaciones en todos los idiomas. Dos revisores independientes seleccionaron los artículos elegibles y extrajeron los datos de los artículos seleccionados. Con base en los 405 artículos encontrados, luego de aplicar los criterios de inclusión, se incluyeron 28 artículos. En vista de las consecuencias de la privación del sueño, se encontraron factores de riesgo de sobrepeso y obesidad, mayores resultados de las mediciones antropométricas (circunferencia de cintura, circunferencia de cadera y porcentaje de grasa corporal), mayor índice de masa corporal, mayor consumo calórico, se encontraron mayores ingestas de procesados alimentos y menor ingesta de frutas. Se concluye que la privación del sueño tiene consecuencias negativas para los niños y adolescentes, teniendo como uno de los principales resultados el riesgo de sobrepeso y obesidad, mayor ingesta de alimentos procesados y más calóricos, y menor ingesta de frutas. Dados estos hallazgos, la educación para la salud sobre este tema debe ser discutida más a fondo entre los padres y / o tutores de niños y adolescentes.
\end{abstract}

Palabras clave: Privación del sueño; Niños; Adolescentes.

\title{
1. Introdução
}

O sono é um processo biológico complexo e essencial, uma necessidade diária para todos os seres humanos, independente de idade, sexo ou origem étnica. Além de manter o funcionamento normal do cérebro, o sono tem papel importante no controle das funções dos outros sistemas do corpo, e isso se torna muito evidente nos estados de privação de sono (Aldabal \& Bahammam, 2011).

As evidências sugerem que a duração e a qualidade do sono bem como o sistema circadiano desempenham papéis importantes na regulação metabólica que podem predispor à obesidade (Arora \& Taheri, 2015). Vale ressaltar que, o sono adequado torna-se mais essencial ainda na primeira infância e na infância para o crescimento e o desenvolvimento físico e psicossocial (Agüero \& Rivera, 2016).

A American Academy of Sleep Medicine recomenda que a duração do sono seja de pelo menos nove horas para crianças e oito horas para adolescentes, quem dorme menos que o recomendado é classificado como tendo sono insuficiente (Tambalis, Panagiotakos, Psarra \& Sidossis, 2018). Estudos indicam que as crianças atualmente têm pelo menos 30 minutos a menos de sono por dia do que a quantidade recomendada (Halal et al., 2015).

Pesquisas detectaram privação de sono já na idade pré-escolar, com um declínio esperado na duração do sono de até duas horas e meia na adolescência e um declínio notável no sono aos nove anos (Martinez et al., 2014). Dados de neuroimagem mostram que a privação de sono aumenta a atividade nos centros de recompensa do cérebro em resposta a imagens de comida saborosa, bem como ' desejo de comer' (McDonald et al., 2015).

Evidências apontam que a privação de sono em crianças/adolescentes pode levar a um maior consumo de alimentos não saudáveis (Westerlund, Ray \& Roos, 2009; Garaulet et al., 2011; Tatone-Tokuda et al., 2011; Beebe et al., 2013; Lu et al., 2014; Simon et al., 2015; Mozaffarian et al., 2020).

Diante destas evidências, reunir e analisar artigos já publicados sobre as consequências da privação do sono são importantes para planejamento de políticas públicas neste âmbito. Assim, o objetivo desse trabalho é realizar uma revisão integrativa para analisar as consequências alimentares e nutricionais em crianças e adolescentes que sofrem de privação de sono. 


\section{Metodologia}

Foi realizada uma revisão integrativa seguindo o guideline PRISMA (Moher et al., 2009). A presente investigação foi realizada para responder à pergunta: Quais as consequências alimentares e nutricionais da privação do sono em crianças e adolescentes? Para buscar os artigos relacionados a este tema foram utilizadas as bases de dados PubMed, Biblioteca Virtual da Saúde (BVS) e Web of Science com estudos publicados até 03/12/2020. Não houve restrição data de início da coleta para recuperação do máximo de artigos possível. Foram considerados artigos elegíveis: I) realizados com crianças e/ou adolescentes que possuem privação do sono; II) disponíveis na integra; II) disponível online; IV) em todos os idiomas. Quanto aos critérios de exclusão: I) carta ao editor; II) Revisões sistemáticas, integrativas e narrativas.

Foram empregados descritores, palavras chaves e os operadores booleanos AND e OR para elaboração da estratégia de busca. Em cada base de dados foi elaborada uma estratégia de busca, conforme apresentado no Quadro 1. Os dados obtidos nas bases de dados foram exportados para a plataforma virtual Rayyan QCRI para auxiliar na retirada de duplicações e seleção dos artigos (Ouzzani, Hammady, Fedorowicz \& Elmagarmid, 2016).

No primeiro momento foram feitas leituras de títulos e resumos por dois autores (JPO e RVR). Em caso de desacordo entre os dois revisores, um terceiro autor (NRB) avaliou e, por consenso, foi tomada a decisão final sobre a inclusão ou não do artigo. No segundo momento, os artigos foram lidos na sua integralidade. Após a seleção dos artigos foram extraídas as seguintes variáveis: autores, país, tipo de estudo, tamanho amostral, média de idade (criança ou adolescentes), média de sono e resultados principais.

Quadro 1. Estratégia de busca nas bases de dados PubMed, Biblioteca Virtual da Saúde e Web of Science.

\begin{tabular}{|c|c|}
\hline Base de dados & tratégia de busca \\
\hline $\begin{array}{l}\text { PubMed } \\
318 \text { artigos }\end{array}$ & $\begin{array}{l}\text { "Child"'Mesh] OR (Children) OR (childhood) OR "Adolescent"[Mesh] OR (Adolescents) OR (Adolescence) OR } \\
\text { (Teens) OR (Teen) OR (Teenagers) OR (Teenager) OR (Youth) OR (Youths) OR (Adolescents, Female) OR } \\
\text { (Adolescent, Female) OR (Female Adolescent) OR (Female Adolescents) OR (Adolescents, Male) OR (Adolescent, } \\
\text { Male) OR (Male Adolescent) OR (Male Adolescents) AND "Sleep Deprivation"[Mesh] OR (Deprivation, Sleep) } \\
\text { OR (Deprivations, Sleep) OR (Sleep Deprivations) OR (REM Sleep Deprivation) OR (Deprivation, REM Sleep) OR } \\
\text { (Deprivations, REM Sleep) OR (REM Sleep Deprivations) OR (Sleep Deprivation, REM) OR (Sleep Deprivations, } \\
\text { REM) OR (Sleep Fragmentation) OR (Fragmentation, Sleep) OR (Fragmentations, Sleep) OR (Sleep Fragmentations) } \\
\text { OR (Insufficient Sleep Syndrome) OR (Insufficient Sleep Syndromes) OR (Syndrome, Insufficient Sleep) OR } \\
\text { (Syndromes, Insufficient Sleep) AND "Pediatric Obesity"[Mesh] OR (Obesity, Pediatric) OR (Childhood Onset } \\
\text { Obesity) OR (Obesity, Childhood Onset) OR (Obesity in Childhood) OR (Child Obesity) OR (Obesity, Child) OR } \\
\text { (Childhood Obesity) OR (Obesity, Childhood) OR (Infant Overweight) OR (Overweight, Infant) OR (Infantile } \\
\text { Obesity) OR (Obesity, Infantile) (Infant Obesity) OR (Obesity, Infant) OR (Childhood Overweight) OR (Overweight, } \\
\text { Childhood) OR (Adolescent Overweight) OR (Overweight, Adolescent) OR (Adolescent Obesity) OR (Obesity, } \\
\text { Adolescent) OR (Obesity in Adolescence) OR "Sugar-Sweetened Beverages"[Mesh] OR (Beverage, Sugar- } \\
\text { Sweetened) OR (Beverages, Sugar-Sweetened) OR (Sugar Sweetened Beverages) OR (Sugar-Sweetened Beverage) } \\
\text { OR (Sugar-Added Beverages) OR (Beverage, Sugar-Added) OR (Beverages, Sugar-Added) OR (Sugar Added } \\
\text { Beverages) OR (Sugar-Added Beverage) OR (Sweetened Drinks) OR (Drink, Sweetened) OR (Drinks, Sweetened) } \\
\text { OR (Sweetened Drink) OR (Sugar-Sweetened Soft Drinks) OR (Drink, Sugar-Sweetened Soft) OR (Drinks, Sugar- } \\
\text { Sweetened Soft) OR (Soft Drink, Sugar-Sweetened) OR (Soft Drinks, Sugar-Sweetened) OR (Sugar Sweetened Soft } \\
\text { Drinks) OR (Sugar-Sweetened Soft Drink) OR (Sweetened Beverages) OR (Beverage, Sweetened) OR (Beverages, } \\
\text { Sweetened) OR (Sweetened Beverage) OR (Sugar-Sweetened Sodas) OR (Soda, Sugar-Sweetened) OR (Sodas, Sugar- } \\
\text { Sweetened) OR (Sugar Sweetened Sodas) OR (Sugar-Sweetened Soda) OR "Eating"'[Mesh] OR (Food Intake) OR } \\
\text { (Intake, Food) OR (Ingestion) }\end{array}$ \\
\hline $\begin{array}{l}\text { Biblioteca Virtual } \\
\text { da Saúde (BVS) } \\
\text { Medline (161) } \\
\text { Lilacs (5) } \\
\text { IBESC (2) } \\
\text { BINASC (1) }\end{array}$ & $\begin{array}{l}\text { MH:"Criança" OR (Criança) OR (Child) OR (Niño) OR (Crianças) OR MH:M01.060.406\$ OR MH:"Adolescente" } \\
\text { (Adolescente) OR (Adolescent) OR (Adolescente) OR (adolescentes) OR (Adolescencia) OR (Jovem) OR (Jovens) } \\
\text { OR (Juventude) OR MH:M01.060.057\$ AND MH:"Privação do sono" OR (Privação do sono) OR (Sleep } \\
\text { Deprivation) OR (Privación de Sueño) OR (Fragmentação do Sono) OR (Privação do Sono REM) OR (Síndrome do } \\
\text { Sono Insuficiente) OR MH:C10.886.425.175 OR MH:C23.888.592.796.772 OR MH:F02.830.855.671 OR } \\
\text { MH:F03.870.400.099\$ AND MH:"Obesidade Pediátrica" OR (Obesidade Pediátrica) OR (Pediatric Obesity) OR } \\
\text { (Obesidad Pediátrica) OR (Obesidade Infantil) OR MH:C18.654.726.500.720 OR MH:C23.888.144.699.500.750 OR } \\
\text { MH:E01.370.600.115.100.160.120.699.500.750 OR MH:G07.100.100.160.120.699.500.750\$ OR MH:"Ingestão de } \\
\text { Alimentos" OR (Ingestão de Alimentos) OR (Eating) OR (Ingestión de Alimentos) OR (Ingestão Alimentar) OR } \\
\text { MH: G07.203.650.283 OR MH: G10.261.330 OR MH:"Bebidas Adoçadas com Açúcar" OR (Bebidas Adoçadas } \\
\text { com Açúcar) OR (Sugar-Sweetened Beverages) OR (Bebidas Azucaradas) OR (Bebidas Adoçadas) OR (Bebidas com } \\
\text { Açúcares Adoçados) OR (Refrigerantes Adoçados com Açúcar) OR MH: G07.203.100.772 OR MH:J02.200.772\$ }\end{array}$ \\
\hline
\end{tabular}




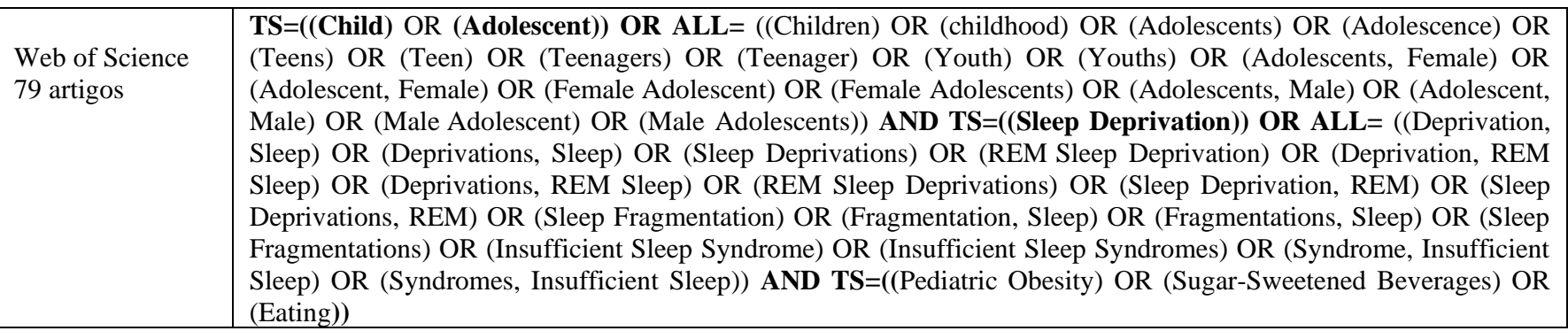

Fonte: Autores.

\section{Resultados}

Foram identificados 405 artigos, sendo que 318 no PubMed, 79 na Web of Science e na BVS 8 artigos. Vale ressaltar que na BVS havia quatros diferentes bases, Medline, Lilacs, IBESC e BINASC.O Medline foi excluído, pois esta base também faz parte do PubMed. Portanto dos 405 artigos, 40 foram inicialmente excluídos por duplicação. Após leitura de título e resumo, selecionou-se 38 artigos para leitura integral. Após análise na íntegra destes artigos, 10 foram excluídos e 28 estudos preencheram os critérios de inclusão na análise (Figura 1).

A exclusão dos 10 artigos após a leitura na íntegra se deu devido aos seguintes motivos: Um a idade dos participantes era acima de 18 anos (Haghighatdoost, Karimi, Esmaillzadeh \& Azadbakht, 2012); Dois eram estudos de revisão (Halal \& Nunes, 2019; Insuga et al., 2013); Dois eram correspondência ao autor; Um ainda está em andamento e não possuiam dados completos (Ward et al., 2019); Quatro não entraram nos critérios de inclusão por não avaliarem os desfechos de interesse da revisão (Bhurosy \& Thiagarajah, 2019; Hager et al., 2016; Ames, Holfeld \& Leadbeater, 2016; Rutters et al., 2010).

A maioria dos estudos foram realizados em países desenvolvidos $78 \%(n=22)$. No que se refere ao tipo de estudo incluído, 39\% $(n=11)$ eram transversais, 39\% $(n=11)$ coorte, $21 \%(n=6)$ intervenção, sendo que dois eram randomizados. O tamanho da população dos estudos variou de 10 a 177.091 participantes. Dentre os estudos analisados $46,4 \%$ ( $\mathrm{n}=13$ ) foram realizados com crianças, $21,4 \%(n=6)$ com adolescentes e 32,1\% ( $n=9)$ com crianças/adolescentes. O estudo que apresentou menor média de sono de crianças foi o de Arora e colaboradores (2018) com 7,6 horas (Arora \& Taheri, 2014). Já o estudo com crianças com maior hora de sono foi de 11,48 horas (McDonald et al., 2015). No que se refere aos estudos realizados com adolescentes, a menor média foi de 5,10 horas e a maior de 9,3 horas (Duraccio, Zaugg \& Jensen, 2019; Beebe et al., 2013) (Tabela 1).

Ao analisar as consequências nutricionais, encontrou-se que $25 \%(n=7)$ dos estudos a privação de sono em crianças e adolescentes foi fator de risco para sobrepeso e obesidade (Arora et al., 2018; Jansen et al., 2018; Chaput et al., 2011; Agüero \& Rivera, 2016; Jong et al., 2012; Wang et al., 2016; Halal et al., 2015).

Também, evidenciou que as medidas antropométricas (Circunferência da Cintura (CC), Circunferência do quadril (CQ) e \% de gordura corporal) eram maiores em participantes que sofriam de privação de sono 25\% (n= 7) (Garaulet et al., 2011; Skidmore et al., 2013; Jarrin, McGrath \& Drake, 2013; Feldner et al., 2015; Hjorth et al., 2015; Arora et al., 2018; Jansen et al., 2018). Além das medidas antropométricas alguns estudos mostraram que participantes com privação de sono tiveram um aumento no índice de massa corporal (IMC) 17\% ( $n=5)$ (Martinez et al., 2014; Jansen et al., 2018; McDonald et al., 2015; Garaulet et al., 2011; Tambalis et al., 2018).

Analisando a média do IMC de quem tem privação de sono é possível constatar que as crianças e adolescentes que possuem privação de sono tem IMC maior do que os que possuem sono longo (Tabela 1). 
Tabela 1. Estudos incluídos que comparam a média de sono de crianças/adolescentes segundo o sono curto e longo.

\begin{tabular}{llll}
\hline Estudo & Criança/Adolescente & Sono curto & Sono longo \\
\hline Javaheri et al. (2011) & Adolescente & Média (\%) de IMC & Média (\%) de IMC \\
\hline Tambalis et al. (2018) & Crianças & $13(21,3)$ & $11(15,3)$ \\
& & M: $19,3(3,6)$ & M: $18,9(3,5)$ \\
\hline Tambalis et al. (2018) & Adolescentes & F: $19,2(3,6)$ & F: $18,8(3,5)$ \\
& & M: $22,1(4,0)$ & M: $21,3(3,8)$ \\
\hline Garaulet et al. (2011) & Adolescentes & F: $20,5(3,3)$ & F: $20,1(3,3)$ \\
\hline
\end{tabular}

IMC: Índice de Massa Corporal; M: Masculino; F: Feminino.

Fonte: Autores.

Observou-se um maior consumo de calorias em participantes que sofriam de privação do sono 14\% (n=4) (Mullins et al., 2016; Simon et al., 2015; Beebe et al., 2013; McDonald et al., 2015). Também foram identificados artigos que apresentaram um consumo maior de produtos industrializados como fast-foods, refrigerantes, bem como de doces e sobremesas $10,7 \%$ ( $\mathrm{n}=3$ ) (Simon et al., 2015; Beebe et al., 2013; Mozaffari et al., 2020; Franckle et al., 2015). No mesmo contexto alguns artigos mostraram uma ingestão menor de frutas e legumes em participantes que sofreram privação de sono 7\% (n=2) (Arora \& Taheri, 2014; Franckle et al., 2015).

Ainda, dietas de baixa qualidade e hábitos alimentares inadequados foram associados à privação de sono dos participantes nos estudos 10\% ( $\mathrm{n}=3$ ) (Garaulet et al., 2011; Cespedes et al., 2015; Tambalis et al., 2018). O estudo de Duraccio e colaboradores (2019) aponta a privação de sono nos participantes como sendo a causa da falta de controle relacionado à alimentação, o que pode aumentar a suscetibilidade de comer em excesso. Em dois dos estudos incluídos apontaram a privação de sono como a causa de níveis HOMA mais altos, diminuição da sensibilidade à insulina em adolescentes, e um maior escore de risco metabólico na metade da infância, bem como níveis mais elevados de certos componentes metabólicos (Javaheri, StorferIsser, Rosen, \& Redline, 2011; Cespedes et al., 2014).

Vale destcar que foram evidenciados benefícios da maior duração de sono, como diminuição da ingestão energética, níveis mais baixos de leptina em jejum, menor consumo de açúcar significativamente associado a um percentual de carboidrato menor e um percentual de gordura mais alto, significativa redução do IMC, níveis mais baixos de todas as variáveis de composição corporal em participantes do sexo masculino (Hart et al., 2013; Martinez et al., 2016; Arora \& Taheri, 2014; Skidmore et al., 2013; Cespedes et al., 2016; Garaulet et al., 2011). 
Research, Society and Development, v. 10, n. 11, e557101119903, 2021

(CC BY 4.0) | ISSN 2525-3409 | DOI: http://dx.doi.org/10.33448/rsd-v10i11.19903

Figura 1: Fluxograma PRISMA.

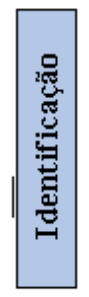

Estudos identificados nas bases de dados eletrônicos

$$
(n=405)
$$

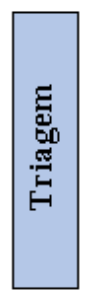

Artigos ap ós retirada da duplicação

$(n=366)$
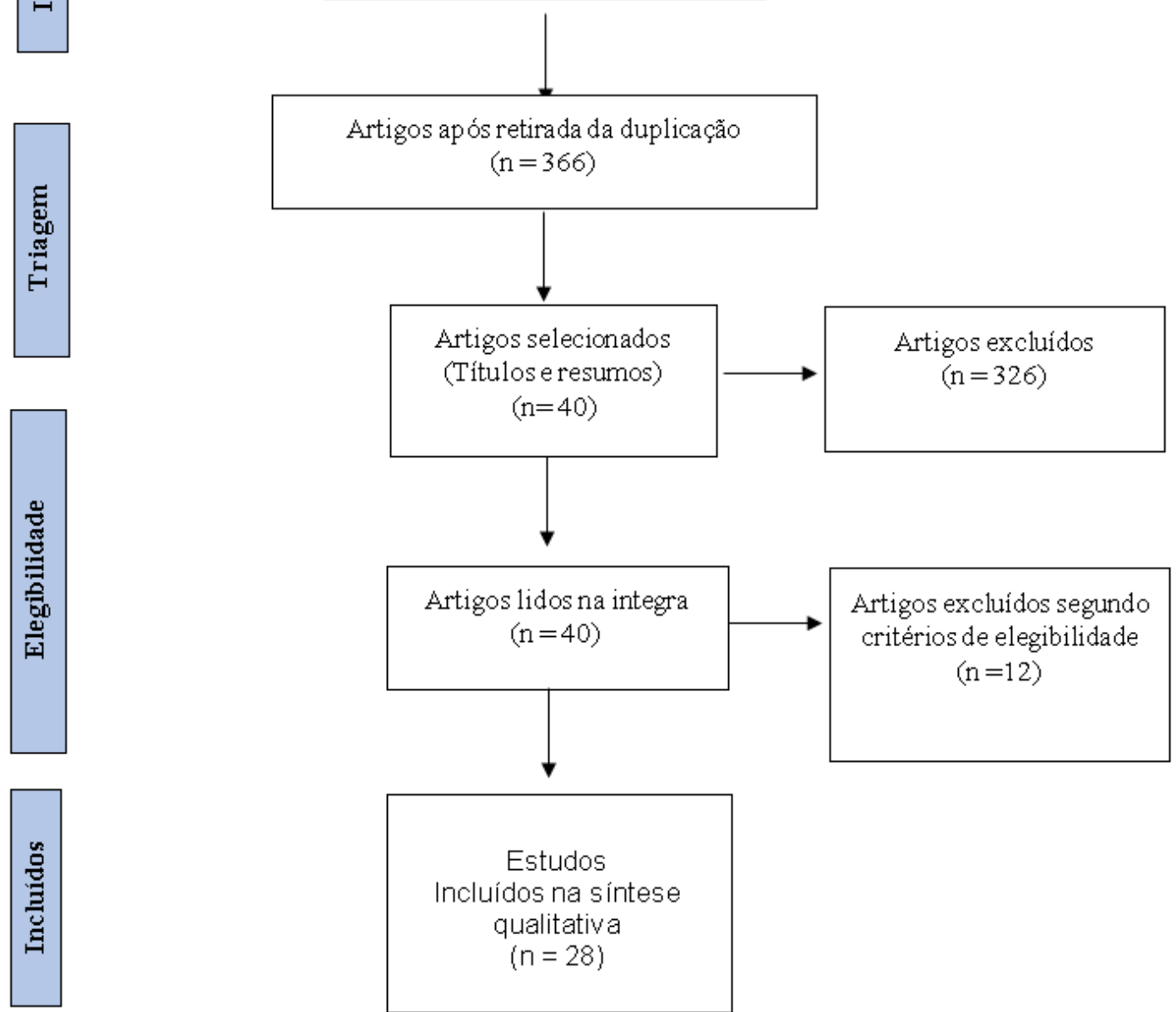

Fonte: Moher D, Liberati A, Tetzlaff J, Altman DG, The PRISMA Group (2009). Preferred Reporting Items for Systematic Reviews and MetaAnalyses: The PRISMA Statement. PLoS Med 6(7): e1000097. doi:10.1371/journal. pmed1000097. For more information, visit: www.prisma-statement.org 
Quadro 2: Informações e características dos estudos incluídos (n=28)

\begin{tabular}{|c|c|c|c|c|c|c|c|}
\hline ID & Autor & País & $\begin{array}{ll}\text { Tipo de } \\
\text { estudo }\end{array}$ & $\begin{array}{l}\text { Tamanho } \\
\text { Amostral }\end{array}$ & Média de idade & Média de sono & Resultados principais \\
\hline 1 & $\begin{array}{l}\text { Javaheri et al. } \\
\text { (2011) }\end{array}$ & EUA & Coorte & 471 & 15,7 anos & $7,63 \pm 1,08$ horas & $\begin{array}{l}\text { 1) Adolescentes que dormiam aprox. } 7,75 \text { h tinham os níveis de HOMA mais } \\
\text { altos previstos, e } 20 \% \text { mais altos nos que dormiam } 5 \text { horas; } \\
\text { 2) Duração de sono cada vez mais curta estão associadas a diminuição da } \\
\text { sensibilidade à insulina em adolescentes. }\end{array}$ \\
\hline 2 & $\begin{array}{lll}\text { Chaput } & \text { et } & \text { al. } \\
(2011) & & \end{array}$ & Canadá & Transversal & 550 & 9,8 anos & $\begin{array}{l}10,8 \quad(\mathrm{DP}=1,4) \text { horas } \\
\text { por noite }\end{array}$ & $\begin{array}{l}\text { Principal descoberta é que a ingestão calórica total e atividade física moderada } \\
\text { e vigorosa não explica a curta conexão sono-obesidade. A curta duração de sono } \\
\text { é um fator de risco para sobrepeso e obesidade em crianças, independente de } \\
\text { covariáveis potenciais. }\end{array}$ \\
\hline 3 & $\begin{array}{l}\text { Garaulet et al. } \\
(2011)\end{array}$ & $\begin{array}{l}\text { Áustria, } \\
\text { Bélgica, } \\
\text { França, } \\
\text { Alemanha, } \\
\text { Grécia, } \\
\text { Hungria, } \\
\text { Itália, } \\
\text { Espanha, } \\
\text { Suécia }\end{array}$ & Transversal & 3.311 & $12,5-17,49$ anos & Média de $8 \mathrm{~h}$. & $\begin{array}{l}\text { 1) Adolescentes com IMC mais baixo apresentaram sono mais longo. A } \\
\text { proporção de adolescentes que comem quantidades adequadas de frutas, } \\
\text { vegetais, peixes, leite desnatado, cereais matinais ou batata frita foi menor entre } \\
\text { aqueles que dormiram menos horas; } \\
\text { 2) Pessoas com sono mais curto tem menor probabilidade de ter hábitos } \\
\text { alimentares adequados; } \\
\text { 3) IMC total e as circunferências da cintura e do quadril se relacionaram } \\
\text { inversamente com a duração do sono em ambos os sexos, enquanto o percentual } \\
\text { de gordura corporal se correlacionou com a duração do sono apenas no sexo } \\
\text { feminino. }\end{array}$ \\
\hline 4 & Jong et al. (2012) & Holanda & Transversal & 4.072 & 4 a 13 anos & $\begin{array}{l}\text { 4-8 anos: } 9-13,25 \mathrm{~h} \\
\text { 9-13 anos: } 8,5-12,5 \mathrm{~h}\end{array}$ & $\begin{array}{l}\text { 1) 4-8 anos: dormindo } 11 \text { horas por noite em comparação com dormir } 12 \text { horas } \\
\text { por noite foi associado ao excesso de peso; } \\
\text { 2) } 9-13 \text { anos: dormindo o } 10,25 \text { h por noite em comparação com dormir x } 11,25 \\
\text { horas por noite foi associada ao excesso de peso. }\end{array}$ \\
\hline 5 & Hart et al. (2013) & Inglaterra & $\begin{array}{l}\text { Ensaio } \\
\text { Clínico }\end{array}$ & 37 & 9,6 anos & 9h:23 min & $\begin{array}{l}\text { Aumento na duração do sono em crianças estão associados a diminuição da } \\
\text { ingestão energética relatada, níveis mais baixos de leptina em jejum e peso } \\
\text { menor. Crianças que dormiram mais tiveram uma redução de } 134 \mathrm{kcal} / \text { dia. }\end{array}$ \\
\hline 6 & Beebe et al. (2013) & EUA & $\begin{array}{l}\text { Ensaio } \\
\text { Clínico }\end{array}$ & 41 & 15,3 anos & $\begin{array}{l}\text { Noites escolares: } 9,3 \mathrm{~h} \\
(\mathrm{DP}: \pm 1,4) \\
\text { Noites não escolares e } \\
7,1 \mathrm{~h}(\mathrm{DP}: \pm 11,2)\end{array}$ & $\begin{array}{l}\text { Durante a restrição de sono os adolescentes consumiram alimentos com IG } \\
\text { significamente mais alto }(\mathrm{p}=0,046) \text { e } \mathrm{CG}(\mathrm{p}=0,037) \text {, e mostraram tendencias de } \\
\text { maior consumo de calorias totais }(\mathrm{p}=0,098) \text { e } \mathrm{CHO}(\mathrm{p}=0,070) \text {. Em geral } \\
\text { adolescentes consumiram uma porção completa de mais doce e sobremesa } \\
\text { durante o final da condição de restrição de sono. }\end{array}$ \\
\hline 7 & Jarrin et al. (2013) & Canadá & Longitudinal & 240 & 12,66 anos & $\begin{array}{l}\text { Durante semana: } \approx 9 \mathrm{~h} \\
\text { Final de semana: } 10 \mathrm{~h}\end{array}$ & $\begin{array}{l}\text { Participantes com distúrbios do sono frequentes tinham CC e CQ e \% de gordura } \\
\text { corporal maior. }\end{array}$ \\
\hline
\end{tabular}




\begin{tabular}{|c|c|c|c|c|c|c|c|}
\hline 8 & $\begin{array}{l}\text { Skidmore et al. } \\
\text { (2013) }\end{array}$ & $\begin{array}{l}\text { Nova } \\
\text { Zelândia }\end{array}$ & Transversal & 685 & 15,8 anos & $\begin{array}{l}\text { Durante a semana: } 9 \\
\text { h:11 min. Finais de } \\
\text { semana: } 10 \mathrm{~h}\end{array}$ & $\begin{array}{l}\text { 1) Maior duração de sono foi associada a níveis mais baixos de todas as variáveis } \\
\text { de composição corporal, incluindo níveis de massa magra, apenas em meninos; } \\
\text { 2) Uma diminuição de uma hora de sono durante a semana foi associada a um } \\
\text { aumento de } 4,5 \% \text { no Índice de massa gorda. }\end{array}$ \\
\hline 9 & Simon et al. (2014) & EUA & $\begin{array}{l}\text { Ensaio } \\
\text { Clínico } \\
\text { Randomizado }\end{array}$ & 31 & 15,7 anos & $\begin{array}{l}\text { Privação de sono: } \\
6,7 \pm 0,6 \mathrm{~h} \\
\text { Sono Habitual: } 9,1 \\
\pm 0,7 \mathrm{~h}\end{array}$ & $\begin{array}{l}\text { Adolescentes comeram } 11 \% \text { mais calorias durante a restrição de sono do que no } \\
\text { sono habitual e } 52 \% \text { mais porções de doces/ sobremesas durante a privação de } \\
\text { sono do que no sono habitual. }\end{array}$ \\
\hline 10 & $\begin{array}{l}\text { Martinez et al. } \\
(2014)\end{array}$ & EUA & $\begin{array}{l}\text { Coorte } \\
\text { longitudinal }\end{array}$ & 229 & 8,8 anos & $\begin{array}{l}\text { 9,5 h: Linha de base } \\
9,6 \mathrm{~h} \text { : Após } 12 \text { meses } \\
9,4 \mathrm{~h}: \text { Após } 24 \text { meses }\end{array}$ & $\begin{array}{l}\text { Crianças com maior duração de sono tiveram um menor IMC e ganho de peso } \\
\text { significativamente menor em } 24 \text { meses. }\end{array}$ \\
\hline 11 & $\begin{array}{lll}\text { Arora } & \text { e Taheri } \\
(2014) & \end{array}$ & Reino Unido & Transversal & 511 & 12,0 anos & $\begin{array}{l}\text { A hora média de dormir } \\
\text { para os cronótipos da } \\
\text { manhã e da noite era } \\
2114 \text { horas e } 2129 \text { horas } \\
(\mathrm{p}=\quad 0,012), \\
\text { respectivamente para } \\
\text { dias de semana. }\end{array}$ & $\begin{array}{l}\text { Jovens com cronótipo noturno tiveram um IMC médio maior em comparação } \\
\text { com os cronótipos matinais. Cronótipos noturnos também consumiram mais } \\
\text { lanches não saudáveis, ingestão inadequada de frutas e vegetais e maior } \\
\text { consumo de cafeína a noite. Cada hora adicional de sono foi associada a uma } \\
\text { pequena, mas significativa redução do IMC. }\end{array}$ \\
\hline 12 & $\begin{array}{l}\text { Cespedes et al. } \\
(2014)\end{array}$ & EUA & $\begin{array}{l}\text { Coorte } \\
\text { prospectivo }\end{array}$ & 652 & 7,9 anos & NI & $\begin{array}{l}\text { A redução do sono desde a primeira infância até a metade da infância foi } \\
\text { associada a um maior escore de risco metabólico na metade da infância, bem } \\
\text { como a níveis mais elevados de certos componentes metabólicos, incluindo } \\
\text { maior CC e insulina. }\end{array}$ \\
\hline 13 & $\begin{array}{l}\text { McDonal et al. } \\
(2014)\end{array}$ & Reino Unido & Coorte & 1278 & $\mathrm{NI}$ & NI & $\begin{array}{l}\text { 1) As crianças do grupo de menor duração de sono }(<10 \mathrm{~h} \text { a noite }) \text { consumiram } \\
\text { em média } 166 \mathrm{Kcal} \text { a noite, enquanto as do grupo de sono mais longo }(>13 \mathrm{~h}) \\
\text { consumiram } 46 \mathrm{kcal} \text {, uma diferença média de } 120 \mathrm{Kcal} \text {; } \\
\text { 2) Crianças que dormem }<10 \mathrm{~h} \text { a noite, o consumo noturno sozinho foi } \\
\text { responsável por } 15,2 \% \text { da ingestão diária de energia. A maioria das calorias } \\
\text { foram obtidas de bebidas, das quais mais de } 98 \% \text { foram bebidas lácteas. }\end{array}$ \\
\hline 14 & $\begin{array}{l}\text { Feldner et al. } \\
\text { (2015) }\end{array}$ & Brasil & Transversal & 41 & 16,61 anos & $\begin{array}{l}\text { Aproximadamente } 7 \\
\text { horas por dia. }\end{array}$ & $\begin{array}{l}\text { 1) } 60,98 \% \text { dos adolescentes obesos possuíam dieta hiperlipidica, } 95,12 \% \text { dieta } \\
\text { normoproteica e } 100 \% \text { dieta normoglicídica; } \\
\text { 2)A má qualidade do sono também apresentou correlação positiva com a massa } \\
\text { corporal. }\end{array}$ \\
\hline 15 & Hjorth et al. (2015) & Dinamarca & Ensaio clínico & 530 & 9,9 anos & $9,16 \pm 0,29$ & $\begin{array}{l}\text { Crianças com sono curto aumentaram a massa gorda em comparação com } \\
\text { crianças com sono longo em } 0,21 \text { (IC: } 95 \% 0,03-0,38) \mathrm{kg} \text {, massa gorda androide } \\
\text { em } 0,02(0,001-0,04) \mathrm{kg} \text {, CC em } 0,73(0,23-1,24) \mathrm{cm} \text {, pressão arterial em } 1,5 \\
(0,4-2,6) \mathrm{mmHg} \text {, ingestão de gordura em } 1,1(0,2-2,0) \text { porcentagem de energia } \\
\text { e diminuiu a atividade física total em } 7,2(1,6-12,7) \% \text { (todos } \mathrm{P} \leq 0,04) \text {. }\end{array}$ \\
\hline 16 & $\begin{array}{l}\text { Mcdonald.et al. } \\
\text { (2015) }\end{array}$ & Reino Unido & Coorte & 1.008 & 5,2 anos & 11,48h (DP: 0,66h) & $\begin{array}{l}\text { Sono mais curto foi associado a um maior IMC e maior responsividade } \\
\text { alimentar. }\end{array}$ \\
\hline
\end{tabular}


Research, Society and Development, v. 10, n. 11, e557101119903, 2021

(CC BY 4.0) | ISSN 2525-3409 | DOI: http://dx.doi.org/10.33448/rsd-v10i11.19903

\begin{tabular}{|c|c|c|c|c|c|c|c|}
\hline 17 & $\begin{array}{l}\text { Franckle } \quad \text { et } \quad \text { al. } \\
(2015)\end{array}$ & EUA & Transversal & 1870 & 10,6 anos & NI & $\begin{array}{l}\text { Participantes que relataram dormir }<10 \mathrm{~h} / \text { dia consumiram refrigerantes com } \\
\text { mais frequência e vegetais com menos frequência em comparação com } \\
\text { participantes que relataram sono ideal. }\end{array}$ \\
\hline 18 & Halal et al. (2015) & Brasil & Coorte & 4.231 & $\begin{array}{l}1,2 \text { e } 4 \text { anos de } \\
\text { idade }\end{array}$ & $\mathrm{NI}$ & $\begin{array}{l}\text { A RP bruta para sobrepeso / obesidade aos } 4 \text { anos de idade entre crianças com } \\
\text { curta duração do sono em qualquer momento entre as idades de } 1 \text { e } 4 \text { anos, em } \\
\text { comparação com aquelas sem curta duração do sono, foi de } 1,26 \text { (IC } 95 \% 0,98 \text { - } \\
1,62 ; ")\end{array}$ \\
\hline 19 & $\begin{array}{l}\text { Cespedes et al. } \\
(2016)\end{array}$ & EUA & Coorte & 1046 & $\begin{array}{l}\text { Primeira infância: } \\
6 \text { meses } \\
\text { Infância: 3,2anos } \\
\text { Meio da Infância: } \\
7,8\end{array}$ & $10,21(2,71-0-13)$ & $\begin{array}{l}\text { 1) Crianças com escores de sono mais altos tinham IMC mais baixo; } \\
\text { 2) Duração insuficiente de sono medida a partir de dados longitudinais da } \\
\text { primeira infância até o meio da infância está associada a uma dieta de baixa } \\
\text { qualidade em crianças. }\end{array}$ \\
\hline 20 & $\begin{array}{l}\text { Agüero e Rivera } \\
\text { (2016) }\end{array}$ & Chile & Transversal & 1810 & 7,7 anos & $\begin{array}{l}\text { Durante Semana: } 9,8( \pm \\
1,0 \mathrm{~h}) \\
\text { Final semana: } 10,8( \pm \\
1,1 \mathrm{~h})\end{array}$ & $\begin{array}{l}\text { 1) Dormem menos que recomendado durante a semana e final de semana: } 49,9 \% \\
\text { e } 16,7 \% \text {, respectivamente. Restrição é maior na semana que no Fim de Semana; } \\
\text { 2) Consomem refrigerante à tarde/noite, } 51,1 \% \text {. Bebem café ou chás antes de } \\
\text { dormir, } 63,2 \% \text {; } \\
\text { 3) Percentual de alunos que dormem menos que o recomendado aumenta com o } \\
\text { aumento da idade e dobra entre } 6 \text { e } 10 \text { anos; } \\
\text { 4) Associação entre menos horas de sono e um risco aumentado de } \\
\text { sobrepeso/obesidade. }\end{array}$ \\
\hline 21 & Wang et al. (2016) & China & Coorte & 48.922 & 3 e 5 anos & $\mathrm{NI}$ & $\begin{array}{l}\text { 1) A incidência de sobrepeso e obesidade em crianças de } 3 \text { a } 5 \text { anos foi de } 9,3 \% \\
\text { e } 2,1 \% \text { respectivamente e maior em meninos. Dormir menos de } 10 \text { h foi } \\
\text { notavelmente associado a um maior risco de peso; } \\
\text { 2) Estado de amamentação das crianças e a idade da mãe no momento do parto } \\
\text { também variaram entre as diferentes categorias de duração do sono. }\end{array}$ \\
\hline 22 & $\begin{array}{l}\begin{array}{l}\text { Mullins } \\
(2016)\end{array} \\
\text { et } \quad \text { al. }\end{array}$ & EUA & $\begin{array}{l}\text { Ensaio } \\
\text { Clínico }\end{array}$ & 10 & $\begin{array}{l}32-47 \text { meses; } 41,2 \\
\text { meses) }\end{array}$ & NI & $\begin{array}{l}\text { Quando em restrição de sono, as crianças consumiram a mais: } 21 \% \text { kcal; } 25 \% \\
\text { açúcar e } 26 \% \text { de CHO. }\end{array}$ \\
\hline 23 & $\begin{array}{l}\text { Martinez et al. } \\
(2016)\end{array}$ & EUA & Coorte & 247 & 10,3 anos. & $9,6(\mathrm{DP}=0,8) \mathrm{h}$ & $\begin{array}{l}\text { A maior duração do sono foi associada ao menor consumo de açúcar, } \\
\text { significativamente associado a um \% CHO inferior e um \% GOR mais alto, } \\
\text { maior consumo de ácido graxo poliinsaturado. As meninas consumiam menos } \\
\text { energia e menos açúcar. }\end{array}$ \\
\hline 24 & Arora et al. (2018) & Catar & Transversal & 335 & 9,0 anos & $\begin{array}{lr}\text { Durante } & \text { semana:7,6 } \\
\pm 0,7 \mathrm{~h} & \\
\text { Fim de } & \text { semana: } \\
8,2 \pm 0,9 \mathrm{~h} & \end{array}$ & $\begin{array}{l}\text { A baixa eficiência do sono foi positivamente associada com o percentual de } \\
\text { gordura corporal. Aqueles que dormiram }<8 \text { h tiveram risco } 4 \text { ou } 5 \text { vezes maior } \\
\text { da doença (obesidade) }\end{array}$ \\
\hline
\end{tabular}


Research, Society and Development, v. 10, n. 11, e557101119903, 2021

(CC BY 4.0) | ISSN 2525-3409 | DOI: http://dx.doi.org/10.33448/rsd-v10i11.19903

\begin{tabular}{|c|c|c|c|c|c|c|c|}
\hline 25 & $\begin{array}{lll}\text { Jansen } & \text { et } & \text { al. } \\
(2018) & & \end{array}$ & México & Coorte & 528 & 14,4 anos & $\begin{array}{l}516 \pm 58 \mathrm{~min} \\
(8,6 \pm 1,0 \mathrm{~h})\end{array}$ & $\begin{array}{l}\text { 1) Participantes com sono insuficientemente teve o maior IMC, \% de gordura } \\
\text { corporal, dobra cutânea tricipital e CC; } \\
\text { 2) Prevalência ajustada de obesidade foi de } 154 \% \text { maior em adolescentes com } \\
\text { sono insuficiente em comparação com aqueles com sono suficiente. }\end{array}$ \\
\hline 26 & $\begin{array}{l}\text { Tambalis et al. } \\
(2018)\end{array}$ & Grécia & Transversal & 177.091 & 9,88 anos & $\begin{array}{l}\text { Durante semana: } 8,6 \\
(1,6) \\
\text { Final de semana: } 9,7 \\
(1,8) \text {. }\end{array}$ & $\begin{array}{l}\text { Participantes que foram classificados como tendo sono insuficiente registraram } \\
\text { aumento de IMC, piores hábitos alimentares em comparação com aqueles com } \\
\text { sono suficiente da mesma faixa etária e sexo. }\end{array}$ \\
\hline 27 & $\begin{array}{l}\text { Duraccio, Zaugg e } \\
\text { Jensen (2019) }\end{array}$ & EUA & $\begin{array}{l}\text { Ensaio } \\
\text { Clínico }\end{array}$ & 64 & 16,25 anos & $\begin{array}{l}5,10 \mathrm{~h} \text { (DP } 1 / 40,35 \text {; faixa } \\
1 / 4 \quad 4,63-5,87 \text { ) em } \\
\text { privação de sono e } \\
\text { média de } 8,99 \text { horas } \\
\text { (DP } 1 / 4 \quad 0,37 ; \text { faixa } 1 / 4 \\
8,34-9,25) \text { em condição } \\
\text { de sono normal. }\end{array}$ & $\begin{array}{l}\text { 1) Adolescentes com restrição de sono demonstram prejuízos no controle } \\
\text { inibitório relacionado a alimentação e aumentos na recompensa alimentar, } \\
\text { independentemente do peso; } \\
\text { 2) Adolescentes com restrição de sono percebem alimentos como mais atraentes, } \\
\text { o que pode aumentar a suscetibilidade a comer em excesso. }\end{array}$ \\
\hline 28 & $\begin{array}{l}\text { Mozaffari et al. } \\
\text { (2019) }\end{array}$ & Irã & Transversal & 14.274 & 12,28 anos & $8,57(1,23)$ horas/dia & $\begin{array}{l}\text { O sono curto em comparação ao sono longo foi associado a maior chance de } \\
\text { consumir lanches salgados, refrigerantes, fast foods, chá e chá com açúcar. }\end{array}$ \\
\hline
\end{tabular}

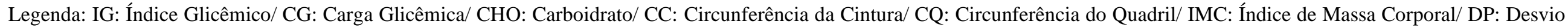
Padrão/ NI: Não Informado/ RP: Razão de Prevalência.

Fonte: Autores. 


\section{Discussão}

A partir dos resultados apresentados foi possível observar que a privação de sono gera consequências nutricionais negativas diretas para a saúde de crianças e adolescentes. Neste sentido, torna-se de grande relevância que os pais/responsáveis saibam da importância do sono para a saúde de seus filhos. Portanto, temas sobre higiene do sono deveriam ser mais abordados em consultas pediátricas. Visto que, a promoção adequada de horas de sono é uma das recomendações para a prevenção da obesidade infantil (Scaraficci et al., 2020).

Dentre as consequências nutricionais devido à privação do sono, o risco para sobrepeso e obesidade foi um dos resultados negativos dessa prática. O estudo de Ruan e colaboradores (2015) corrobora com os resultados da presente pesquisa, no qual realizou-se uma revisão com crianças e adolescentes mostrando que a duração do sono pode estar inversa e longitudinalmente associada ao risco de sobrepeso e obesidade, assim como foi observado no estudo de Li, Zhang, Huang \& Chen (2017) onde a curta duração do sono foi associada a um aumento de $45 \%$ no risco de desenvolver obesidade. Sabe-se que o sono tem efeito benéfico sobre o organismo, e a privação de sono pode desencadear baixos níveis de leptina e altos níveis de grelina, interferindo assim na regulação do apetite (Taheri et al., 2004). Neste sentido, mais tempo acordado implica em mais tempo para se alimentar, o que pode aumentar o consumo calórico contribuindo assim para o excesso peso, e consequentemente aumento do IMC e de medidas antropométricas como circunferência de cintura, de abdômen e percentual de gordura, o qual foi relatado em vários estudos.

Alterações metabólicas também foram relatadas como consequência negativa da privação do sono. Um estudo realizado no Brasil apontou que adolescentes com privação de sono tiveram um índice de sensibilidade a insulina mediana inferior em comparação com os adolescentes que tiveram sono adequado (De Bernardi Rodrigues et al., 2016). Já no estudo de Androutsos et al. (2014), houve um aumento de HOMA IR em crianças de nove a 13 anos com privação de sono, alto tempo de tela e alto consumo de bebidas açucaradas. Outro resultado semelhante foi no estudo de Matthews e colaboradores (2012) que mostraram que a curta duração do sono durante a semana e a curta duração geral do sono foram associadas ao aumento do HOMA-IR após o ajuste para sexo, idade, raça, circunferência da cintura z- escores e resíduos de IMC, enquanto a maior duração do sono nos finais de semana não foi associada ao HOMA-IR. Esses resultados consolidam a presente investigação, pois, evidenciam como a privação do sono afeta o metabolismo da glicose negativamente.

Outra consequência foi aumento do consumo calórico que também foi notado nas crianças / adolescentes que sofrem de privação de sono. Este aumento calórico durante a privação do sono também foi observado em indivíduos adultos, além de redução no consumo de frutas e vegetais, aumento no consumo de refrigerantes e fast-food (Baron, Reid, Kern \& Zee, 2011). Comer em excesso após a perda de sono pode representar uma resposta compensatória homeostática, que provavelmente é produzida para compensar o déficit de energia que resulta da privação de sono (Hogenkamp et al., 2013). No entanto Morselli e colaboradores (2010) relatam que a insulina e a leptina parecem modular negativamente o sistema de recompensa alimentar, enquanto a grelina exerce efeitos opostos, as mudanças nessas concentrações hormonais após a restrição do sono podem contribuir para o aumento da alimentação hedônica, em vez de homeostática, que pode ser típica de pessoas que dormem pouco. Mudanças na atividade cerebral associadas à recompensa alimentar foram observadas após a privação de sono (Yang, Schnepp, \& Tucker, 2019). Esse aumento no consumo de produtos industrializados implica em uma alimentação de baixa qualidade e acaba gerando hábitos alimentares inadequados.

No presente estudo a menor média de sono das crianças foi de 7,6 horas e a maior 11,48 horas. Já nos adolescentes a menor média de sono foi de 5,10 horas e a maior média de 9,3 horas. Segundo a Academia Americana de Medicina do Sono recomenda que crianças de um a dois anos de idade devem dormir de 11 a 14 horas (incluindo cochilos), crianças de três a cinco 
Research, Society and Development, v. 10, n. 11, e557101119903, 2021

(CC BY 4.0) | ISSN 2525-3409 | DOI: http://dx.doi.org/10.33448/rsd-v10i11.19903

anos de idade devem dormir de dez a 13 horas (incluindo cochilos), crianças de seis a 12 anos de idade devem dormir nove a 12 horas e adolescentes de 13 a 18 anos devem dormir de oito a dez horas (Paruthi et al., 2016). Diante disso, nota-se que as crianças e adolescentes estão dormindo menos que o recomendado.

Diante desses achados é importante ressaltar que a má higiene do sono pode ser fator desencadeante neste cenário de privação de sono, com o uso de telas (celular, tablet, videogame, TV) no quarto. Estudos recentes demonstraram que a exposição à luz de intensidade relativamente baixa pode alterar os ritmos circadianos e suprimir a secreção noturna de melatonina (Cain \& Gradisar, 2010; Owens, Adolescent Sleep Working Group, \& Committee on Adolescence, 2014; Perrault et al., 2019). Além disso, o uso de cafeína e refrigerantes a noite, trabalhos escolares, e a falta de monitoramento dos pais ou regras sobre a hora de dormir.

Essa investigação descreve consequências importantes devido à privação do sono em crianças e adolescentes, porém algumas limitações são identificadas. A primeira delas é possível que algum artigo não tenha sido recuperado pela estratégia de busca, visto que, o desfecho avaliado (consequências nutricionais) é um termo amplo. Outra limitação foi a forma de avaliação do sono, visto que, alguns artigos a avaliação foram feitos através de relatos dos pais e não por actigrafia, o que poderia influenciar na duração exata do sono.

\section{Conclusão}

O presente trabalho evidenciou que a privação de sono traz consequências negativas para essa faixa etária, tendo como um dos resultados principais o risco para sobrepeso e obesidade, maior ingestão de alimentos industrializados, mais calóricos e menor ingestão de frutas. Já a duração do sono adequada apresentou benefícios para a saúde das crianças e adolescentes. Tais achados, podem subsidiar discussões entre pesquisadores/profissionais de saúde para posterior elaboração de diretrizes e/ou material educativo para orientação adequada do sono de crianças/adolescentes.

Ressalta-se também a importância de mais estudos sobre a privação de sono de crianças/adolescentes e suas consequências, visto que, pesquisas neste sentido podem identificar os principais fatores que podem desencadear a privação do sono, bem como, subsidiar programas de intervenções com os pais e ou responsáveis para orientações sobre este tema.

\section{Referências}

Agüero, S. D., \& Rivera, P. H. (2016). Association between the amount of sleep and obesity in Chilean schoolchildren. Archivos argentinos de pediatria, 114(2), 114-119. https://doi.org/10.5546/aap.2016.eng.114

Aldabal, L., \& Bahammam, A. S. (2011). Metabolic, endocrine, and immune consequences of sleep deprivation. The open respiratory medicine journal, 5, 3143. https://doi.org/10.2174/1874306401105010031

Ames, M. E., Holfeld, B., \& Leadbeater, B. J. (2016). Sex and age group differences in the associations between sleep duration and BMI from adolescence to young adulthood. Psychology \& health, 31(8), 976-992. https://doi.org/10.1080/08870446.2016.1163360

Androutsos, O., Moschonis, G., Mavrogianni, C., Roma-Giannikou, E., Chrousos, G. P., Kanaka-Gantenbein, C., \& Manios, Y. (2014). Identification of lifestyle patterns, including sleep deprivation, associated with insulin resistance in children: the Healthy Growth Study. European journal of clinical nutrition, 68(3), 344-349. https://doi.org/10.1038/ejen.2013.280

Arora, T., \& Taheri, S. (2014). Associations among late chronotype, body mass index and dietary behaviors in young adolescents. International journal of obesity (2005), 39(1), 39-44. https://doi.org/10.1038/ijo.2014.157

Arora, T., Gad, H., Omar, O. M., Choudhury, S., Chagoury, O., Sheikh, J., \& Taheri, S. (2018). The associations among objectively estimated sleep and obesity indicators in elementary schoolchildren. Sleep medicine, 47, 25-31. https://doi.org/10.1016/j.sleep.2018.03.014

Baron, K. G., Reid, K. J., Kern, A. S., \& Zee, P. C. (2011). Role of sleep timing in caloric intake and BMI. Obesity (Silver Spring, Md.), 19(7), 1374-1381. https://doi.org/10.1038/oby.2011.100

Beebe, D. W., Simon, S., Summer, S., Hemmer, S., Strotman, D., \& Dolan, L. M. (2013). Dietary intake following experimentally restricted sleep in adolescents. Sleep, 36(6), 827-834. https://doi.org/10.5665/sleep.2704 
Research, Society and Development, v. 10, n. 11, e557101119903, 2021 (CC BY 4.0) | ISSN 2525-3409 | DOI: http://dx.doi.org/10.33448/rsd-v10i11.19903

Bhurosy, T., \& Thiagarajah, K. (2020). Are Eating Habits Associated with Adequate Sleep among High School Students?. The Journal of school health, 90(2), 81-87. https://doi.org/10.1111/josh.12852

Cain, N., \& Gradisar, M. (2010). Electronic media use and sleep in school-aged children and adolescents: A review. Sleep medicine, 11(8), 735-742. https://doi.org/10.1016/j.sleep.2010.02.006

Cespedes, E. M., Hu, F. B., Redline, S., Rosner, B., Gillman, M. W., Rifas-Shiman, S. L., \& Taveras, E. M. (2016). Chronic insufficient sleep and diet quality: Contributors to childhood obesity. Obesity (Silver Spring, Md.), 24(1), 184-190. https://doi.org/10.1002/oby.21196

Cespedes, E. M., Rifas-Shiman, S. L., Redline, S., Gillman, M. W., Peña, M. M., \& Taveras, E. M. (2014). Longitudinal associations of sleep curtailment with metabolic risk in mid-childhood. Obesity (Silver Spring, Md.), 22(12), 2586-2592. https://doi.org/10.1002/oby.20894

Chaput, J. P., Lambert, M., Gray-Donald, K., McGrath, J. J., Tremblay, M. S., O'Loughlin, J., \& Tremblay, A. (2011). Short sleep duration is independently associated with overweight and obesity in Quebec children. Canadian journal of public health = Revue canadienne de sante publique, 102(5), 369-374. https://doi.org/10.1007/BF03404179

Duraccio, K. M., Zaugg, K., \& Jensen, C. D. (2019). Effects of Sleep Restriction on Food-Related Inhibitory Control and Reward in Adolescents. Journal of pediatric psychology, 44(6), 692-702. https://doi.org/10.1093/jpepsy/jsz008

Feldner, C. B., Freitas, Z. R. R. de, Rodrigues, B. S., Nunes, J. E. D., Santos, H. C. \& Cheik, N. C. (2015). Associação da ingestão de macronutrientes com o padrão de sono de adolescentes obesos. ConScientiae Saúde, 14(4), 592-599.

Franckle, R. L., Falbe, J., Gortmaker, S., Ganter, C., Taveras, E. M., Land, T., \& Davison, K. K. (2015). Insufficient sleep among elementary and middle school students is linked with elevated soda consumption and other unhealthy dietary behaviors. Preventive medicine, 74, 36-41. https://doi.org/10.1016/j.ypmed.2015.02.007

Garaulet, M., Ortega, F. B., Ruiz, J. R., Rey-López, J. P., Béghin, L., Manios, Y., Cuenca-García, M., Plada, M., Diethelm, K., Kafatos, A., Molnár, D., AlTahan, J., \& Moreno, L. A. (2011). Short sleep duration is associated with increased obesity markers in European adolescents: effect of physical activity and dietary habits. The HELENA study. International journal of obesity (2005), 35(10), 1308-1317. https://doi.org/10.1038/ijo.2011.149

Hager, E. R., Calamaro, C. J., Bentley, L. M., Hurley, K. M., Wang, Y., \& Black, M. M. (2016). Nighttime Sleep Duration and Sleep Behaviors among Toddlers from Low-Income Families: Associations with Obesogenic Behaviors and Obesity and the Role of Parenting. Childhood obesity (Print), 12(5), 392-400. https://doi.org/10.1089/chi.2015.0252

Haghighatdoost, F., Karimi, G., Esmaillzadeh, A., \& Azadbakht, L. (2012). Sleep deprivation is associated with lower diet quality indices and higher rate of general and central obesity among young female students in Iran. Nutrition (Burbank, Los Angeles County, Calif.), 28(11-12), 1146-1150. https://doi.org/10.1016/j.nut.2012.04.015

Halal, C., \& Nunes, M. L. (2019). Sleep and weight-height development. Jornal de pediatria, 95 Suppl 1, 2-9. https://doi.org/10.1016/j.jped.2018.10.009

Halal, C., Matijasevich, A., Howe, L. D., Santos, I. S., Barros, F. C., \& Nunes, M. L. (2016). Short Sleep Duration in the First Years of Life and Obesity/Overweight at Age 4 Years: A Birth Cohort Study. The Journal of pediatrics, 168, 99-103.e3. https://doi.org/10.1016/j.jpeds.2015.09.074

Hart, C. N., Carskadon, M. A., Considine, R. V., Fava, J. L., Lawton, J., Raynor, H. A., Jelalian, E., Owens, J., \& Wing, R. (2013). Changes in children's sleep duration on food intake, weight, and leptin. Pediatrics, 132(6), e1473-e1480. https://doi.org/10.1542/peds.2013-1274

Hjorth, M. F., Sjödin, A., Dalskov, S. M., Damsgaard, C. T., Michaelsen, K. F., Biltoft-Jensen, A., Andersen, R., Ritz, C., Chaput, J. P., \& Astrup, A. (2016). Sleep duration modifies effects of free ad libitum school meals on adiposity and blood pressure. Applied physiology, nutrition, and metabolism $=$ Physiologie appliquee, nutrition et metabolisme, 41(1), 33-40. https://doi.org/10.1139/apnm-2015-0319

Hogenkamp, P. S., Nilsson, E., Nilsson, V. C., Chapman, C. D., Vogel, H., Lundberg, L. S., Zarei, S., Cedernaes, J., Rångtell, F. H., Broman, J. E., Dickson, S. L., Brunstrom, J. M., Benedict, C., \& Schiöth, H. B. (2013). Acute sleep deprivation increases portion size and affects food choice in young men. Psychoneuroendocrinology, 38(9), 1668-1674. https://doi.org/10.1016/j.psyneuen.2013.01.012

Insuga, V. S., Merino, M., Pozo, R. L. del., Mota, C. C. de la, Villena, A. P. \& Álvarez, M. P. (2013). Sueño y obesidad en la infancia. Acta Pediátrica Española, 71(9), 191-198.

Jansen, E. C., Dunietz, G. L., Chervin, R. D., Baylin, A., Baek, J., Banker, M., Song, P., Cantoral, A., Tellez Rojo, M. M., \& Peterson, K. E. (2018). Adiposity in Adolescents: The Interplay of Sleep Duration and Sleep Variability. The Journal of pediatrics, 203, 309-316. https://doi.org/10.1016/j.jpeds.2018.07.087

Jarrin, D. C., McGrath, J. J., \& Drake, C. L. (2013). Beyond sleep duration: distinct sleep dimensions are associated with obesity in children and adolescents. International journal of obesity (2005), 37(4), 552-558. https://doi.org/10.1038/ijo.2013.4

Javaheri, S., Storfer-Isser, A., Rosen, C. L., \& Redline, S. (2011). Association of short and long sleep durations with insulin sensitivity in adolescents. The Journal of pediatrics, 158(4), 617-623. https://doi.org/10.1016/j.jpeds.2010.09.080

Jiang F. (2017). Sleep duration and adiposity in early adolescents: response. Journal of public health (Oxford, England), 39(1), 203. https://doi.org/10.1093/pubmed/fdw003

Jong, E. de, Stocks, T., Visscher, T. L., HiraSing, R. A., Seidell, J. C., \& Renders, C. M. (2012). Association between sleep duration and overweight: the importance of parenting. International journal of obesity (2005), 36(10), 1278-1284. https://doi.org/10.1038/ijo.2012.119

Kawada T. (2017). Sleep duration and adiposity in early adolescents. Journal of public health (Oxford, England), 39(1), 202. https://doi.org/10.1093/pubmed/fdw002 
Research, Society and Development, v. 10, n. 11, e557101119903, 2021 (CC BY 4.0) | ISSN 2525-3409 | DOI: http://dx.doi.org/10.33448/rsd-v10i11.19903

Li, L., Zhang, S., Huang, Y., \& Chen, K. (2017). Sleep duration and obesity in children: A systematic review and meta-analysis of prospective cohort studies. Journal of paediatrics and child health, 53(4), 378-385. https://doi.org/10.1111/jpc.13434

Lu, Q., Hou, F., Sun, Y., Zhang, Z., \& Tao, F. (2014). Zhonghua liu xing bing xue za zhi = Zhonghua liuxingbingxue zazhi, 35(4), 381-385.

Martinez, S. M., Tschann, J. M., Greenspan, L. C., Deardorff, J., Penilla, C., Flores, E., Pasch, L. A., Gregorich, S. E., \& Butte, N. F. (2014). Is it time for bed? Short sleep duration increases risk of obesity in Mexican American children. Sleep medicine, 15(12), 1484-1489. https://doi.org/10.1016/j.sleep.2014.09.009

Martinez, S. M., Tschann, J. M., Butte, N. F., Gregorich, S. E., Penilla, C., Flores, E., Greenspan, L. C., Pasch, L. A., \& Deardorff, J. (2017). Short Sleep Duration Is Associated With Eating More Carbohydrates and Less Dietary Fat in Mexican American Children. Sleep, 40(2), zsw057. https://doi.org/10.1093/sleep/zsw057

Matthews, K. A., Dahl, R. E., Owens, J. F., Lee, L., \& Hall, M. (2012). Sleep duration and insulin resistance in healthy black and white adolescents. Sleep, 35(10), 1353-1358. https://doi.org/10.5665/sleep.2112

McDonald, L., Wardle, J., Llewellyn, C. H., Johnson, L., van Jaarsveld, C. H., Syrad, H., \& Fisher, A. (2015). Sleep and nighttime energy consumption in early childhood: a population-based cohort study. Pediatric obesity, 10(6), 454-460. https://doi.org/10.1111/ijpo.12006

McDonald, L., Wardle, J., Llewellyn, C. H., \& Fisher, A. (2015). Nighttime sleep duration and hedonic eating in childhood. International journal of obesity (2005), 39(10), 1463-1466. https://doi.org/10.1038/ijo.2015.132

Moher, D., Liberati, A., Tetzlaff, J., Altman, D. G., \& PRISMA Group (2009). Preferred reporting items for systematic reviews and meta-analyses: the PRISMA statement. Annals of internal medicine, 151(4), 264-W64. https://doi.org/10.7326/0003-4819-151-4-200908180-00135

Morselli, L., Leproult, R., Balbo, M., \& Spiegel, K. (2010). Role of sleep duration in the regulation of glucose metabolism and appetite. Best practice \& research. Clinical endocrinology \& metabolism, 24(5), 687-702. https://doi.org/10.1016/j.beem.2010.07.005

Mozaffarian, N., Heshmat, R., Ataie-Jafari, A., Motlagh, M. E., Ziaodini, H., Shafiee, G., Taheri, M., Mansourian, M., Qorbani, M., \& Kelishadi, R. (2020). Association of sleep duration and snack consumption in children and adolescents: The CASPIAN-V study. Food science \& nutrition, 8(4), 1888-1897. https://doi.org/10.1002/fsn3.1471

Mullins, E. N., Miller, A. L., Cherian, S. S., Lumeng, J. C., Wright, K. P., Jr, Kurth, S., \& Lebourgeois, M. K. (2017). Acute sleep restriction increases dietary intake in preschool-age children. Journal of sleep research, 26(1), 48-54. https://doi.org/10.1111/jsr.12450

Ouzzani, M., Hammady, H., Fedorowicz, Z., \& Elmagarmid, A. (2016). Rayyan-a web and mobile app for systematic reviews. Systematic reviews, 5(1), 210. https://doi.org/10.1186/s13643-016-0384-4

Owens, J., Adolescent Sleep Working Group, \& Committee on Adolescence (2014). Insufficient sleep in adolescents and young adults: an update on causes and consequences. Pediatrics, 134(3), e921-e932. https://doi.org/10.1542/peds.2014-1696

Paruthi, S., Brooks, L. J., D'Ambrosio, C., Hall, W. A., Kotagal, S., Lloyd, R. M., Malow, B. A., Maski, K., Nichols, C., Quan, S. F., Rosen, C. L., Troester, M. M., \& Wise, M. S. (2016). Consensus Statement of the American Academy of Sleep Medicine on the Recommended Amount of Sleep for Healthy Children: Methodology and Discussion. Journal of clinical sleep medicine : JCSM : official publication of the American Academy of Sleep Medicine, 12(11), 1549-1561. https://doi.org/10.5664/jcsm.6288

Perrault, A. A., Bayer, L., Peuvrier, M., Afyouni, A., Ghisletta, P., Brockmann, C., Spiridon, M., Hulo Vesely, S., Haller, D. M., Pichon, S., Perrig, S., Schwartz, S., \& Sterpenich, V. (2019). Reducing the use of screen electronic devices in the evening is associated with improved sleep and daytime vigilance in adolescents. Sleep, 42(9), zsz125. https://doi.org/10.1093/sleep/zsz125

Rodrigues, A. M. De B., da Silva, C., Vasques, A. C., Camilo, D. F., Barreiro, F., Cassani, R. S., Zambon, M. P., Antonio, M. Â., Geloneze, B., \& Brazilian Metabolic Syndrome Study (BRAMS) Investigators (2016). Association of Sleep Deprivation With Reduction in Insulin Sensitivity as Assessed by the Hyperglycemic Clamp Technique in Adolescents. JAMA pediatrics, 170(5), 487-494. https://doi.org/10.1001/jamapediatrics.2015.4365

Ruan, H., Xun, P., Cai, W., He, K., \& Tang, Q. (2015). Habitual Sleep Duration and Risk of Childhood Obesity: Systematic Review and Dose-response Metaanalysis of Prospective Cohort Studies. Scientific reports, 5, 16160. https://doi.org/10.1038/srep16160

Rutters, F., Gerver, W. J., Nieuwenhuizen, A. G., Verhoef, S. P., \& Westerterp-Plantenga, M. S. (2010). Sleep duration and body-weight development during puberty in a Dutch children cohort. International journal of obesity (2005), 34(10), 1508-1514. https://doi.org/10.1038/ijo.2010.161

Scaraficci, A. C., Piantamar, J. P. S., Tanimoto, R. M. F., Martis, V. M. B. \& Stuchi-Perez, E. G. (2020). Obesidade Infantil: Recomendações para orientações inicial. CuidArte. Enfermagem, 2(14), 257-263.

Simon, S. L., Field, J., Miller, L. E., DiFrancesco, M., \& Beebe, D. W. (2015). Sweet/dessert foods are more appealing to adolescents after sleep restriction. PloS one, 10(2), e0115434. https://doi.org/10.1371/journal.pone.0115434

Skidmore, P. M., Howe, A. S., Polak, M. A., Wong, J. E., Lubransky, A., Williams, S. M., \& Black, K. E. (2013). Sleep duration and adiposity in older adolescents from Otago, New Zealand: relationships differ between boys and girls and are independent of food choice. Nutrition journal, 12, 128 . https://doi.org/10.1186/1475-2891-12-128

Taheri, S., Lin, L., Austin, D., Young, T., \& Mignot, E. (2004). Short sleep duration is associated with reduced leptin, elevated ghrelin, and increased body mass index. PLoS medicine, 1(3), e62. https://doi.org/10.1371/journal.pmed.0010062 
Research, Society and Development, v. 10, n. 11, e557101119903, 2021 (CC BY 4.0) | ISSN 2525-3409 | DOI: http://dx.doi.org/10.33448/rsd-v10i11.19903

Tambalis, K. D., Panagiotakos, D. B., Psarra, G., \& Sidossis, L. S. (2018). Insufficient Sleep Duration Is Associated With Dietary Habits, Screen Time, and Obesity in Children. Journal of clinical sleep medicine : JCSM : official publication of the American Academy of Sleep Medicine, 14(10), 1689-1696. https://doi.org/10.5664/jcsm.7374

Tatone-Tokuda, F., Dubois, L., Ramsay, T., Girard, M., Touchette, E., Petit, D., \& Montplaisir, J. Y. (2012). Sex differences in the association between sleep duration, diet and body mass index: a birth cohort study. Journal of sleep research, 21(4), 448-460. https://doi.org/10.1111/j.1365-2869.2011.00989.x

Taveras, E. M., Gillman, M. W., Peña, M. M., Redline, S., \& Rifas-Shiman, S. L. (2014). Chronic sleep curtailment and adiposity. Pediatrics, 133(6), 10131022. https://doi.org/10.1542/peds.2013-3065

Wang, F., Liu, H., Wan, Y., Li, J., Chen, Y., Zheng, J., Huang, T., \& Li, D. (2016). Sleep Duration and Overweight/Obesity in Preschool-Aged Children: A Prospective Study of up to 48,922 Children of the Jiaxing Birth Cohort. Sleep, 39(11), 2013-2019. https://doi.org/10.5665/sleep.6234

Ward, A. L., Galland, B. C., Haszard, J. J., Meredith-Jones, K., Morrison, S., McIntosh, D. R., Jackson, R., Beebe, D. W., Fangupo, L., Richards, R., Te Morenga, L., Smith, C., Elder, D. E., \& Taylor, R. W. (2019). The effect of mild sleep deprivation on diet and eating behaviour in children: protocol for the Daily Rest, Eating, and Activity Monitoring (DREAM) randomized cross-over trial. BMC public health, 19(1), 1347. https://doi.org/10.1186/s12889-019-7628-x

Westerlund, L., Ray, C., \& Roos, E. (2009). Associations between sleeping habits and food consumption patterns among 10-11-year-old children in Finland. The British journal of nutrition, 102(10), 1531-1537. https://doi.org/10.1017/S0007114509990730

Yang, C. L., Schnepp, J., \& Tucker, R. M. (2019). Increased Hunger, Food Cravings, Food Reward, and Portion Size Selection after Sleep Curtailment in Women Without Obesity. Nutrients, 11(3), 663. https://doi.org/10.3390/nu11030663 